\title{
Water Quality Evaluation of Aquifers Around Igboukwu and Environs, Southeastern Nigeria
}

\author{
Ajanobi, C. C., Nfor, B. N.*, Okpoko, E. I. and Anene, C. Z. \\ Department of Geology, Faculty of Physical Sciences, Chukwuemeka Odumegwu Ojukwu University, Nigeria \\ *Corresponding author's email: nforbng@ yahoo.com; Phone no.: +2348034737791
}

\begin{abstract}
Water quality (physico-chemical and biological) of the aquifers of the Tertiary formation of Igboukwu and environs was evaluated using the World Health Organization (WHO) and Nigeria Standard for Drinking Water Quality guidelines with water samples collected from twenty boreholes during pumping tests. The study area covers a total surface area of $450.56 \mathrm{~km}^{2}$ including towns like, Igboukwu, Ezinifite, Uga, Ekwulobia, Nanka, Oko, Aguluezechukwu, Umuchu, Achina, Ekwulumili and Ndiowu. Results showed that the area is underlain by two geological Units, Imo Shale and Nanka Sands, from which the aquifers were tapped. Analysis of the results of physical parameters showed that temperature is ambient, water is colourless, odourless and tasteless for all samples studied. Results of the water chemistry, however showed marked variations; $\mathrm{pH}$ values ranged from 5.20 at Ihuowelle Igboukwu to 6.80, at Ebele Achina (highest), total dissolved solids (TDS) from $11.20 \mathrm{mg} / \mathrm{l}$ at Ezinifite to $71.40 \mathrm{mg} /$ at Achina, turbidity nil, major cations and anions, calcium $\left(\mathrm{Ca}^{2+}\right)$ ranged from $5.0 \mathrm{mg} / \mathrm{l}$ at Okwuchiezeada Ezinifite to $42 \mathrm{mg} / \mathrm{l}$ at Ndiowu, magnesium $\left(\mathrm{Mg}^{2+}\right)$ ranged from $0.01 \mathrm{mg} / \mathrm{l}$ at $\mathrm{Uga}$ to $0.18 \mathrm{mg} / \mathrm{l}$ in Ekwulobia. Iron ion values ranged from $0.02 \mathrm{mg} / \mathrm{l}$ to $0.35 \mathrm{mg} / \mathrm{l}$ in Igboukwu, Umueze Uga $(0.35 \mathrm{mg} / \mathrm{l})$, manganese, copper and residual chlorine were not detected; while Bicarbonate $\left(\mathrm{HCO}_{3}\right)$ ranged from $7.00 \mathrm{mg} / \mathrm{l}$ at Ezinifite to $58.00 \mathrm{mg} / \mathrm{l}$ at $\mathrm{Uga}$ and Sulphate $\left(\mathrm{SO}_{4}{ }^{2}\right)$ ranged from $0.00 \mathrm{mg} / \mathrm{l}$ at Igboukwu to $7.00 \mathrm{mg} / \mathrm{l}$ at Ekwulobia, sulphate ions were not detected in Ekwoulobia, while in Umunze and $\mathrm{Uga}, \mathrm{Mn}^{2+}, \mathrm{Ca}^{2+}$ and $\mathrm{Cl}^{-}$ions were not detected either. Bacteriological results showed that total coliform and fecal coliform were zero. These results thus showed that the aquifers in the study areas contain portable water for domestic use if the wells are well constructed and harnessed.
\end{abstract}

Original Research Article

Received: $11^{\text {th }}$ Feb., 2018 Accepted: 05 ${ }^{\text {th }}$ Mar., 2018

Published: $14^{\text {th }}$ Mar., 2018

Keywords: Water quality Physico-chemical Biological Pumping tests Igboukwu

\section{INTRODUCTION}

$\mathrm{T}$ The project area is located within Anambra State, Southeastern Nigeria and lies within latitudes $5^{\circ} 560^{1}$ and $6^{\circ} 40^{1} \mathrm{~N}$ and longitude, $7^{\circ} 00^{1}$ and $7^{\circ} 100^{1} \mathrm{E}$, encompassing Aguata, Aniocha and Orumba Local Government Area. These areas hydrologically fallwithin the Anambra Basin and more specifically within the Anambra River Basin (Fig. 1). The area hasabundant surface water and groundwater resources.
The main river systems in the study areas are Odo, Obizi, Otalulu, Iyiagu and Uchu.

\section{Geology and hydrogeology}

The Anambra basin is one of the major inland sedimentary basins in Nigeria, which is bounded on the east by the Abakaliki anticlinorium and on the southwesterly direction by the Benin hinge-line, the limits of the Eocene growth faults of the Niger Delta. These growth faults extend from the Calabar hinge-line to the 
Benin hinge-line through the Onitsha high. The basin is about $300 \mathrm{~km}$ long in a northern southwest direction, extending between the Onitsha and environs in Anambra State and Lokoja area in Benue State. Its southwestern extreme is about $48 \mathrm{~km}$ wide (Whiteman, 1982) (fig. 1). The stratigraphic succession in the Anambra Basin and environs have been discussed (Reyment, 1965; Whiteman, 1982; Hoque and Nwajide,
1984).A generalized geologic map of the basin adapted from the geological map of Nigeria by the Geological Survey of Nigeria (1974) is as shown in fig. 2. The geologic cross section shows that the study area is underlain by the Ameki Formation and the Imo Shale. The population here gets its water supplies from boreholes that tap from these two formations as well as from springs and streams.

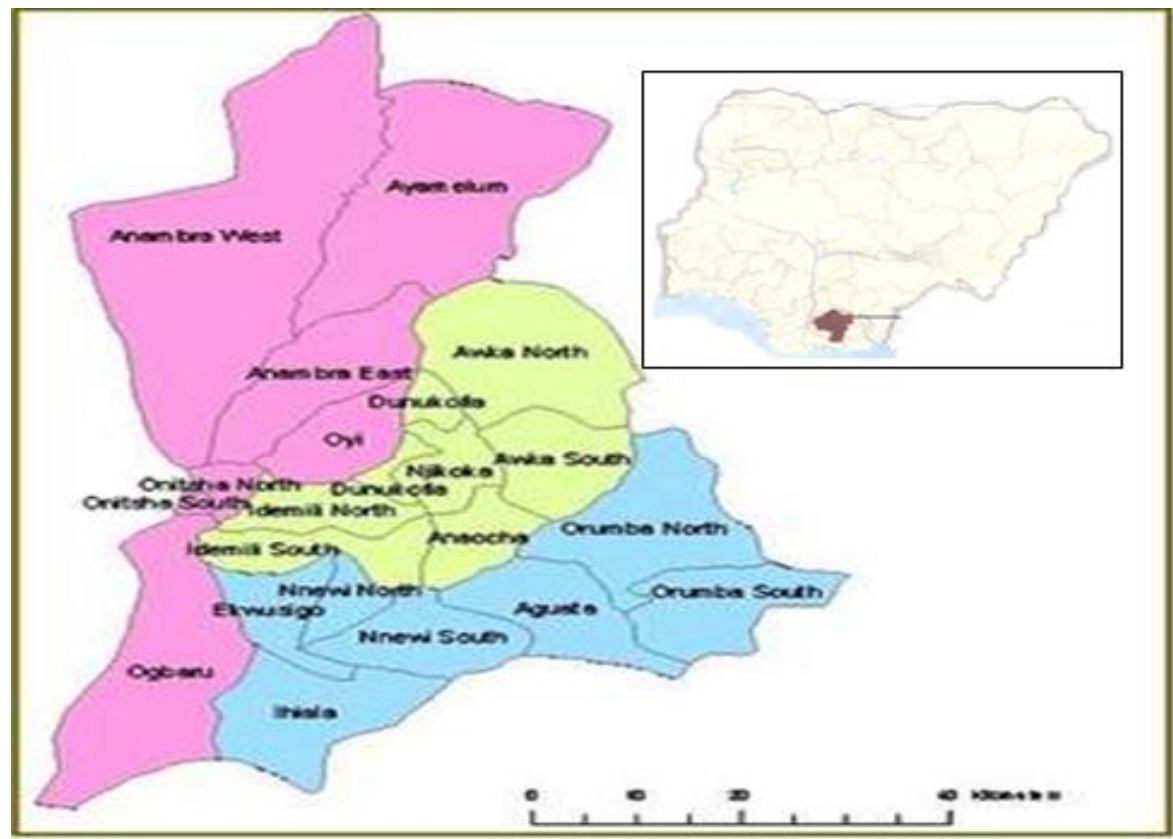

Figure 1: Map of Nigeria Showing the Study Area (Egboka et al, 1993).
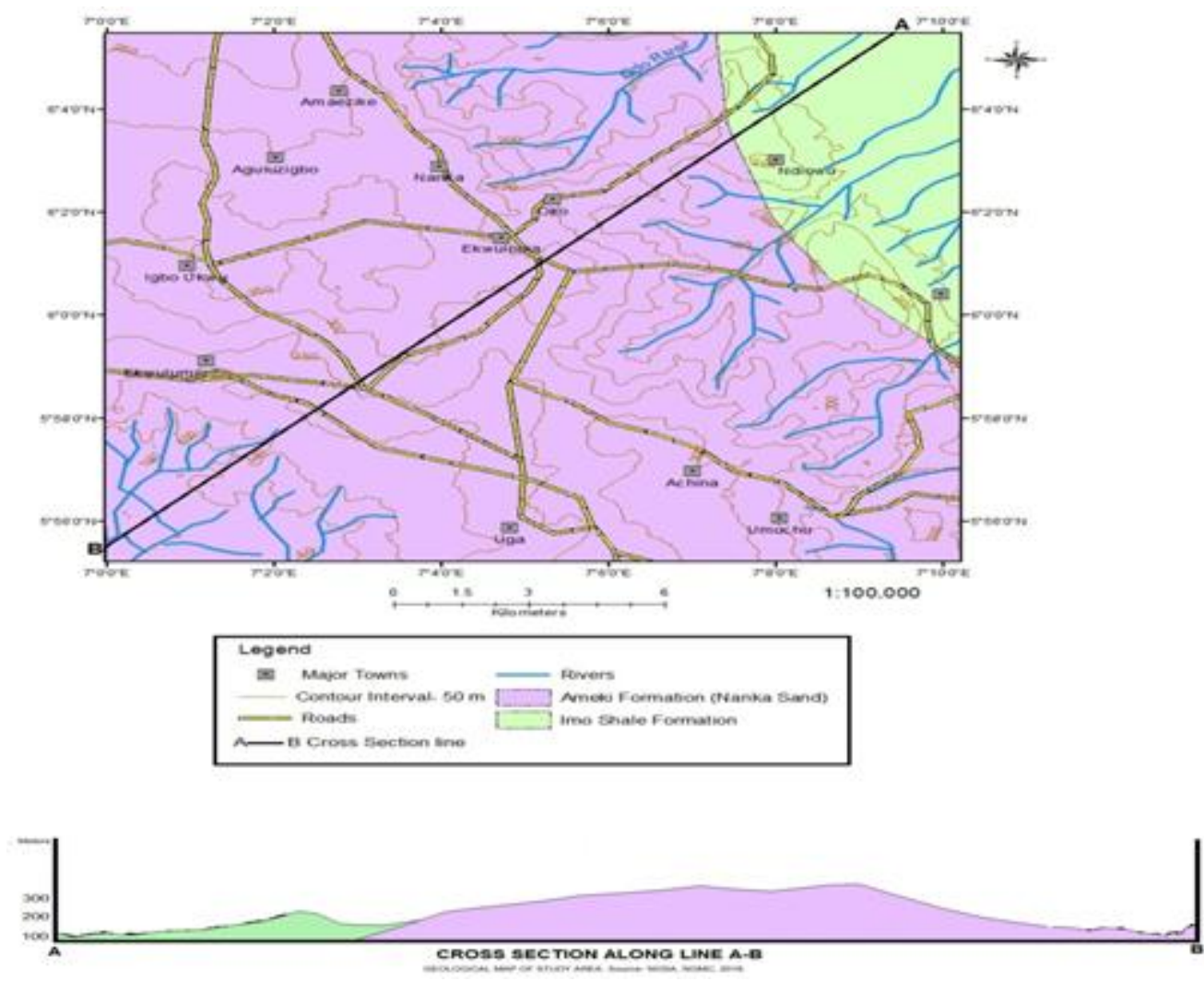

Figure 2: Geological Map of the Study Area (Abor, sheet 300). 
The study area has an undulating topography, with a maximum elevation of $300 \mathrm{~m}$ above sea level and a minimum elevation of $100 \mathrm{~m}$ above sea level. The major surface water body found in the study area is the Orashi River. Other surface waters in the area include otalulu, Uchu, Odor, Streams and the Obizi spring at Ekwulimi. Perennial and seasonal streams, springs drain the area.
There is connectivity existing between surface water systems and groundwater systems; the groundwater systems are recharged as a result of infiltration of parts of overland flow into the soil (Fetter, 2001; Heath, 1983). Figure 3 showed the drainage and location of the study area.
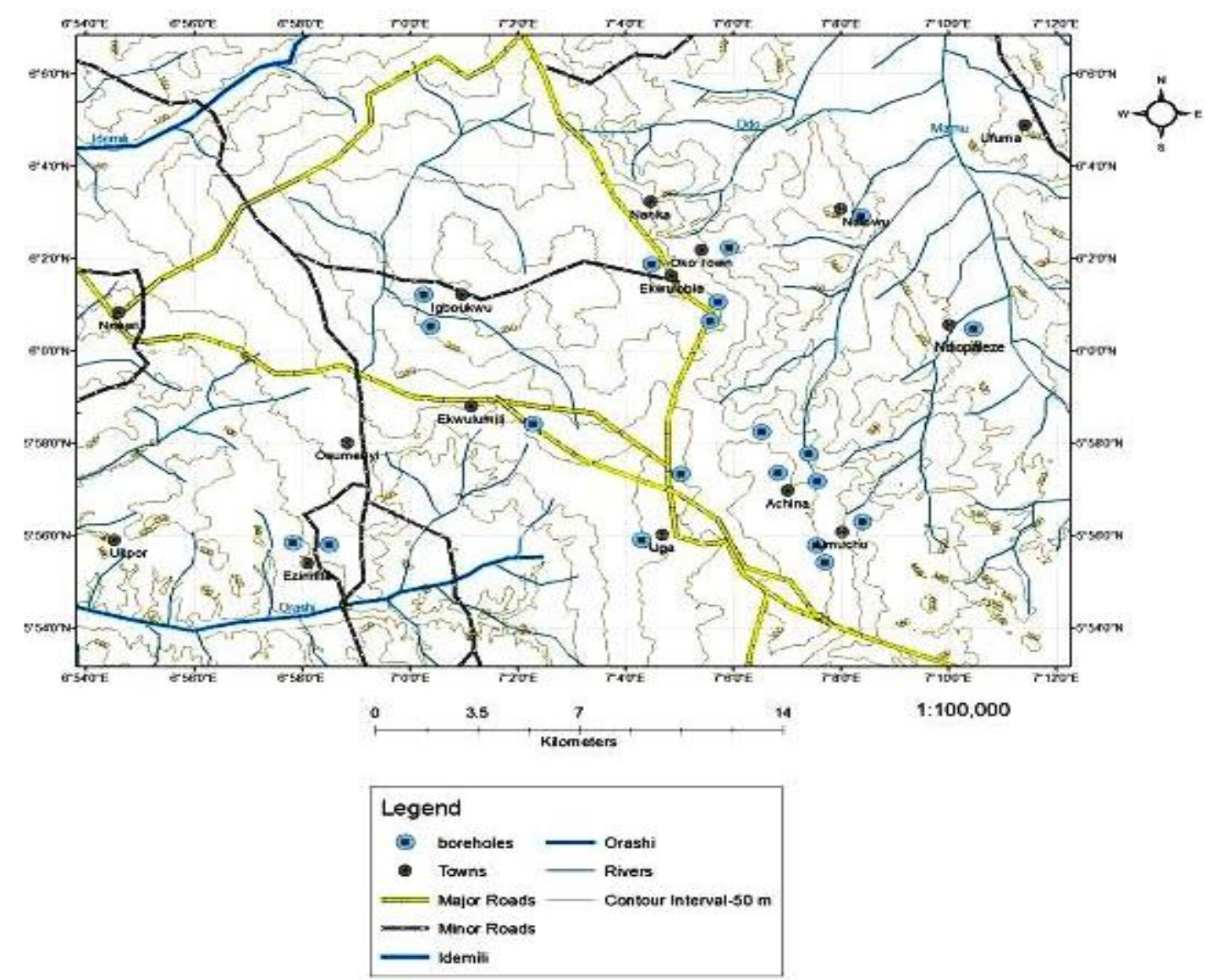

Figure 3: Drainage and Location Map of the Study Area (Adapted from Abor Map Sheet 300).

\section{Climate and Vegetation}

The study area lies within the humid tropical rain climate of Nigeria, with rainfall amounting to $2000 \mathrm{~mm}$ per annum. In Aguata, the average annual temperature is $25.7^{\circ} \mathrm{C}$ and an annual average rainfall of $1975 \mathrm{~mm}$ (Fig. 4). The area experiences two Climatic seasons; the rainy season (March to October) and the dry season (October to March). These seasons have variable regimes. The dry season has the coldest, direst windy period known as the harmathan between November and February. The rainy season has its peaks in July and September with a dip in August known as "the August break". Much rainfall occurs as violent downpours, accompanied by lightning and thunderstorms, heavy flooding, soil leaching, sheet out wash and ground water infiltration and percolation. Minimum temperature is $23^{\circ} \mathrm{C}$, maximum temperature is $34^{\circ} \mathrm{C}$ while the average temperature $28^{\circ} \mathrm{C}$. Relative humidity is $80 \%$ during the raining season and $65 \%$ during the dry season (Okpoko, 1986).The driest month is December, with $11 \mathrm{~mm}$ of rain. The greatest amount of precipitation occurs in September, with an average of $318 \mathrm{~mm}$. March is the warmest month of the year. The temperature in average is $27.4^{\circ} \mathrm{C}$. The lowest average temperatures in the year occur in August, when it is around $24.0^{\circ} \mathrm{C}$. There is a difference of $307 \mathrm{~mm}$ of precipitation between the driest and wettest months. The variation in temperatures throughout the year is 3.4 ${ }^{\circ} \mathrm{C}$.

The vegetation is characteristics of the humid tropical rainforest belt type of Nigeria, with trees, evergreen leaves and thick undergrowth. The vegetation cover has greatly reduced to Savannah type. It is characterized by open vegetative lowland interspersed with tall oil palm trees and deciduous trees. Agriculture practices are intensive, particularly along the hill slopes, dry river valleys and low-lying plains. These, further, reduce the vegetation to the aforesaid Savannah type. In some places, the vegetation has been cleared for agriculture, road construction, industrial sites and urban development. 


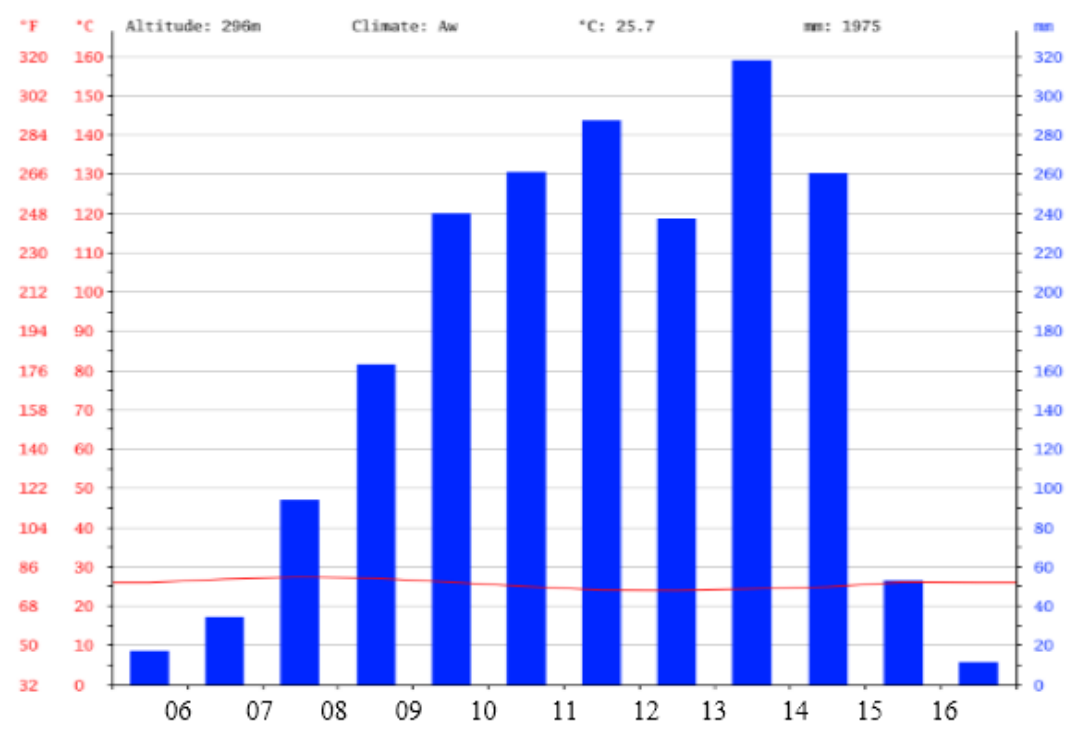

Figure 4: Climate of the study area (Macmillian, 2007).

\section{MATERIALS AND METHODS}

In order to achieve the goals of this research, the materials and methods used were; topographic map of the area on scales of 1:100,000, 1:250,000 and 1:500,000, global positioning system (GPS), plastic containers, generating set, and water depth well sounder.

During standard pumping tests, twenty borehole water samples were collected for geochemical analysis. All the collected samples were carefully labeled according to the respective locations and put in an ice packed bag. The samples where properly labeled indicating the time of collection.

\section{RESULTS AND DATA ANALYSES}

Water quality was assessed using physical, chemical and biological parameters. The physical parameters investigated include appearance, temperature, colour, odour and turbidity. The chemical parameter investigated include $\mathrm{pH}$, conductivity, total dissolve solids, salinity, copper, nitrite, manganese and the major cations and anions. The bacteriological parameters investigated were for fecal coliform count and total coliform. The results were presented in Tables 1 and 2. All investigations/assessment were carried out using standard recommended methodologies and interpreted against World Health Organization (WHO, 2001) and Nigeria Standard for Drinking Water Quality, recommendations/guidelines (NSDWQ, 2007).

Table 1: Statistical Analysis for Major Cations and Anions in Study Areas.

\begin{tabular}{|c|c|c|c|c|c|c|c|}
\hline & Parameter & Mean & $\begin{array}{l}\text { Standard } \\
\text { Deviation }\end{array}$ & Variance & Range & Max. & Mim. \\
\hline \multirow{4}{*}{ 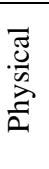 } & $\mathrm{pH}$ & 5.80 & 0.26 & 0.07 & 0.80 & 6.30 & 5.50 \\
\hline & Conductivity $(\mu \mathrm{s} / \mathrm{cm})$ & 67.13 & 29.10 & 844.00 & 104.90 & 141.50 & 36.60 \\
\hline & $\mathrm{TDS}(\mathrm{mg} / \mathrm{l})$ & 35.06 & 14.30 & 205.00 & 52.40 & 70.70 & 18.30 \\
\hline & Hardness (mg/l) & 36.70 & 15.40 & 238.00 & 54.00 & 67.00 & 13.00 \\
\hline \multirow{5}{*}{ ڤ్ల } & Calcium $\left(\mathrm{Ca}^{2+}\right) \mathrm{mg} / \mathrm{l}$ & 24.20 & 9.30 & 87.30 & 33.00 & 41.00 & 8.00 \\
\hline & $\begin{array}{l}\text { Magnesium } \quad\left(\mathrm{mg}^{2+}\right) \\
\mathrm{mg} / \mathrm{l}\end{array}$ & 15.80 & 6.30 & 39.30 & 21.00 & 29.00 & 8.00 \\
\hline & Sodium $\left(\mathrm{Na}^{+}\right) \mathrm{mg} / \mathrm{l}$ & 22.0262 & 8.40 & 70.8215 & 28.842 & 367094 & 7.8674 \\
\hline & Potassium $\left(\mathrm{K}^{+}\right) \mathrm{mg} / \mathrm{l}$ & 8.83 & 2.30 & 5.5099 & 8.29 & 13.05 & 4.76 \\
\hline & $\begin{array}{l}\text { Bicarbonate }\left(\mathrm{HCo}_{3}{ }^{-}\right) \\
\mathrm{mg} / \mathrm{l}\end{array}$ & 35.65 & 20.00 & 392.00 & 72.00 & 80.00 & 8.00 \\
\hline \multirow{2}{*}{$\cdot \frac{0}{0}$} & Sulphate $\left(\mathrm{So}_{4}^{2}\right) \mathrm{mg} / \mathrm{l}$ & 1.75 & 1.60 & 2.70 & 6.00 & 7.00 & 1.00 \\
\hline & $\mathrm{Cl}$ (Chloride)mg/l & 4.9926 & 1.6484 & 2.7173 & 5.8212 & 7.9561 & 2.1349 \\
\hline
\end{tabular}


Table 2: Results Microbiological Analyses.

\begin{tabular}{|c|c|c|c|c|c|c|c|c|c|c|c|c|c|c|c|c|c|c|c|c|c|c|c|c|c|c|}
\hline & \multicolumn{6}{|c|}{ PHYSICAL PARAMETERS } & \multicolumn{18}{|c|}{ CHEMICAL PARAMETERS } & \multicolumn{2}{|c|}{$\begin{array}{l}\text { BACTERIOLO } \\
\text { GICAL } \\
\text { PARAMETERS }\end{array}$} \\
\hline & 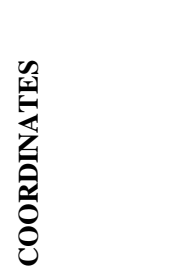 & 秃 & 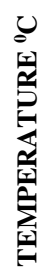 & 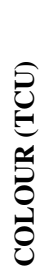 & 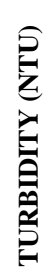 & $\begin{array}{l}\text { 茨 } \\
\text { อิ }\end{array}$ & 竞 & 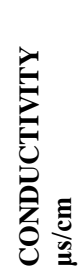 & 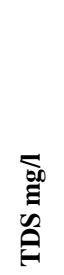 & 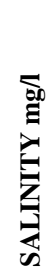 & 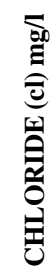 & 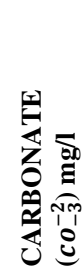 & 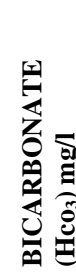 & 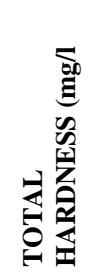 & 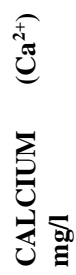 & 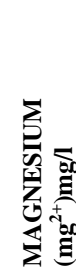 & 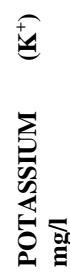 & 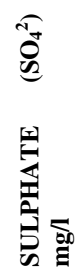 & 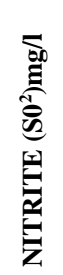 & 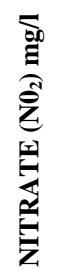 & 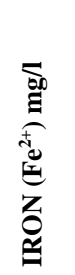 & 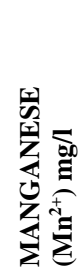 & 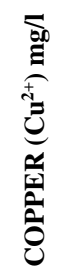 & 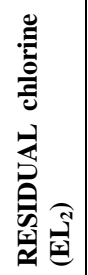 & 这 & 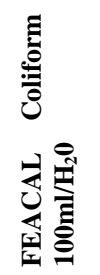 \\
\hline $\begin{array}{r}\text { 1. Ihuowelle- } \\
\text { Igboukwu }\end{array}$ & $\begin{array}{l}\text { N06.01004 } \\
\text { E007.00594 }\end{array}$ & $\frac{\vec{\Xi}}{\tilde{U}}$ & 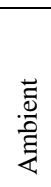 & Nil & Nil & Nil & 5.7 & 28.6 & 14.3 & Nil & Nil & Nil & 9.0 & 40 & 18 & 10 & 8.0 & Nil & 10.5 & 0.03 & 4.0 & 0.21 & 0.03 & - & 0 & 0 \\
\hline $\begin{array}{l}\text { 2. Umume } \\
\text { Umudege- } \\
\text { Igboukwu }\end{array}$ & $\begin{array}{l}\text { N06,00234, } \\
\text { E007.2921 }\end{array}$ & $\frac{\vec{d}}{\tilde{U}}$ & 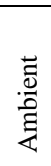 & Nil & Nil & Nil & 5.8 & 31.4 & 15.7 & Nil & $\mathrm{Nil}$ & Nil & 10 & 31 & 9.0 & 22 & - & Nil & Nil & Nil & 0.27 & Nil & Nil & - & 0 & 0 \\
\hline $\begin{array}{l}\text { 3. Okwuchi } \\
\text { Ezeada- } \\
\text { Ezinifite }\end{array}$ & 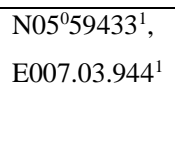 & $\frac{\vec{\Xi}}{U}$ & 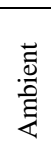 & Nil & Nil & $\mathrm{Nil}$ & 5.8 & 22.3 & 11.3 & Nil & $\mathrm{Nil}$ & Nil & 7.0 & 15 & 5.0 & 10 & - & 7.0 & 0.01 & 1.76 & 0.03 & Nil & Nil & - & 0 & 0 \\
\hline $\begin{array}{l}\text { 4. Ezihe } \\
\text { Umueze } \\
\text { Uga }\end{array}$ & $\begin{array}{l}\text { N05 }^{0} 55.304^{1} \\
\text { E007 } 57.402^{1}\end{array}$ & $\begin{array}{l}\vec{z} \\
\frac{0}{U}\end{array}$ & 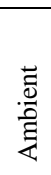 & 90 & 17 & Nil & 5.9 & 38.4 & 19.2 & Nil & $\mathrm{Nil}$ & Nil & 58 & 19 & 7.0 & 12 & - & 2.0 & Nil & Nil & 0.35 & 0.01 & Nil & - & 1 & 1 \\
\hline
\end{tabular}


Table 2: (Continued).

\begin{tabular}{|c|c|c|c|c|c|c|c|c|c|c|c|c|c|c|c|c|c|c|c|c|c|c|c|c|c|c|}
\hline & \multicolumn{6}{|c|}{ PHYSICAL PARAMETERS } & \multicolumn{18}{|c|}{ CHEMICAL PARAMETERS } & \multicolumn{2}{|c|}{$\begin{array}{c}\text { BACTERIOLO } \\
\text { GICAL } \\
\text { PARAMETERS }\end{array}$} \\
\hline & 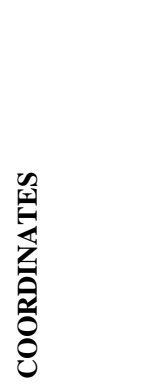 & 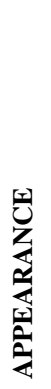 & 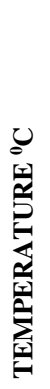 & 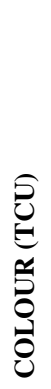 & 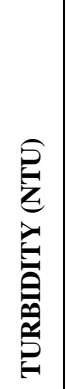 & 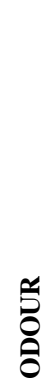 & $\underline{a}$ & 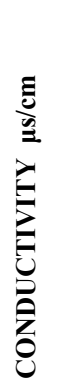 & 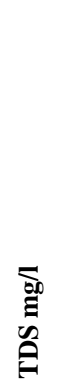 & 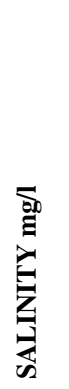 & 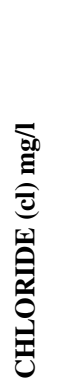 & 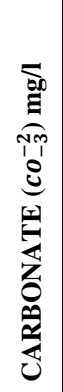 & 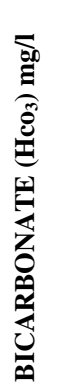 & 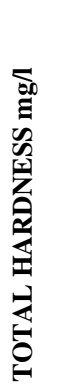 & 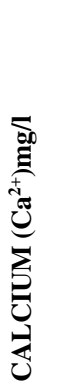 & 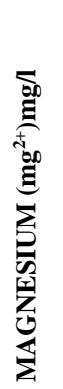 & 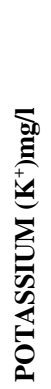 & 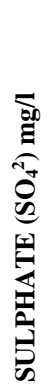 & 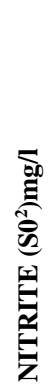 & 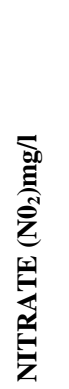 & 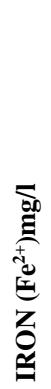 & 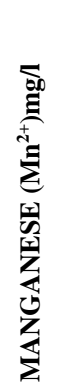 & 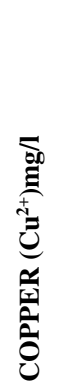 & 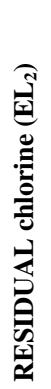 & 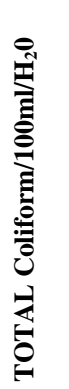 & 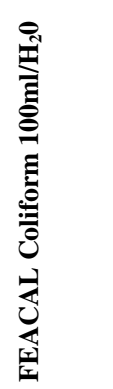 \\
\hline $\begin{array}{l}5 . \\
\text { AgbakoAwar } \\
\text { asi }\end{array}$ & $\begin{array}{l}\text { N05.95515 } \\
\text { E007.0833 } \\
8^{0}\end{array}$ & $\frac{\vec{\varpi}}{U}$ & : & $\begin{array}{l}\mathrm{Ni} \\
1\end{array}$ & $\begin{array}{l}\mathrm{Ni} \\
1\end{array}$ & $\begin{array}{l}\mathrm{Ni} \\
1\end{array}$ & $\begin{array}{l}5.8 \\
0\end{array}$ & 33.50 & $\begin{array}{l}16.7 \\
0\end{array}$ & Nil & Nil & $\begin{array}{l}\mathrm{Ni} \\
1\end{array}$ & 7.00 & $\begin{array}{l}12.0 \\
0\end{array}$ & 7.00 & 5.00 & - & $\begin{array}{l}3.0 \\
0\end{array}$ & Nil & 4.40 & $\begin{array}{l}0.2 \\
0\end{array}$ & Nil & $\begin{array}{l}\mathrm{Ni} \\
1\end{array}$ & - & 6.00 & 0.00 \\
\hline $\begin{array}{l}\text { 6. Progressive } \\
\text { Primary } \\
\text { School } \\
\text { Achina }\end{array}$ & $\begin{array}{l}\text { N05.95299 } \\
0, \\
\text { E007.1222 } \\
1^{0}\end{array}$ & 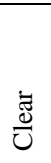 & 苛 & $\begin{array}{l}\mathrm{Ni} \\
1\end{array}$ & $\begin{array}{l}\mathrm{Ni} \\
1\end{array}$ & $\begin{array}{l}\mathrm{Ni} \\
1\end{array}$ & $\begin{array}{l}6.4 \\
5\end{array}$ & $\begin{array}{l}142.5 \\
0\end{array}$ & $\begin{array}{l}71.4 \\
0\end{array}$ & $\begin{array}{l}16.5 \\
0\end{array}$ & $\begin{array}{l}10.0 \\
0\end{array}$ & $\begin{array}{l}\mathrm{Ni} \\
1\end{array}$ & $\begin{array}{l}79.0 \\
0\end{array}$ & $\begin{array}{l}86.0 \\
0\end{array}$ & $\begin{array}{l}52.0 \\
0\end{array}$ & $\begin{array}{l}34.0 \\
0\end{array}$ & - & $\begin{array}{l}2.0 \\
0\end{array}$ & $\begin{array}{l}0.0 \\
2\end{array}$ & 4.40 & $\begin{array}{l}0.2 \\
0\end{array}$ & $\begin{array}{l}0.0 \\
1\end{array}$ & $\begin{array}{l}\mathrm{Ni} \\
1\end{array}$ & - & 3.00 & 0.00 \\
\hline $\begin{array}{l}\text { 7. Umuezeiyi } \\
\text { Community, } \\
\text { Achina }\end{array}$ & $\begin{array}{l}\text { N05.96211 } \\
0, \\
\text { E007.1220 } \\
0^{0}\end{array}$ & 苞 & 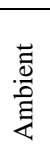 & $\begin{array}{l}\mathrm{Ni} \\
1\end{array}$ & $\begin{array}{l}\mathrm{Ni} \\
1\end{array}$ & $\begin{array}{l}\mathrm{Ni} \\
1\end{array}$ & $\begin{array}{l}6.3 \\
5\end{array}$ & 58.70 & $\begin{array}{l}29.3 \\
0\end{array}$ & Nil & Nil & $\begin{array}{l}\mathrm{Ni} \\
1\end{array}$ & $\begin{array}{l}35.0 \\
0\end{array}$ & $\begin{array}{l}32.0 \\
0\end{array}$ & $\begin{array}{l}19.0 \\
0\end{array}$ & $\begin{array}{l}13.0 \\
0\end{array}$ & - & $\begin{array}{l}1.0 \\
0\end{array}$ & $\begin{array}{l}0.0 \\
1\end{array}$ & $\begin{array}{l}12.0 \\
0\end{array}$ & $\begin{array}{l}0.0 \\
2\end{array}$ & Nil & $\begin{array}{l}\mathrm{Ni} \\
1\end{array}$ & - & 1.00 & 0.00 \\
\hline $\begin{array}{l}\text { 8. Okpo } \\
\text { Ekwulobia }\end{array}$ & $\begin{array}{l}\text { N06.01008 } \\
0 \\
\text { E007.0911 } \\
0\end{array}$ & $\frac{\bar{\Xi}}{\tilde{\Xi}}$ & 节 & $\begin{array}{l}\mathrm{Ni} \\
1\end{array}$ & $\begin{array}{l}\mathrm{Ni} \\
1\end{array}$ & $\begin{array}{l}\mathrm{Ni} \\
1\end{array}$ & $\begin{array}{l}5.9 \\
0\end{array}$ & 50.30 & $\begin{array}{l}25.2 \\
0\end{array}$ & Nil & Nil & $\begin{array}{l}\mathrm{Ni} \\
1\end{array}$ & $\begin{array}{l}57.0 \\
0\end{array}$ & $\begin{array}{l}17.0 \\
0\end{array}$ & $\begin{array}{l}11.0 \\
0\end{array}$ & 6.00 & - & $\begin{array}{l}6.0 \\
0\end{array}$ & Nil & 3.50 & Nil & $\begin{array}{l}0.0 \\
1\end{array}$ & - & - & 2.00 & 0.00 \\
\hline $\begin{array}{l}\text { 9. Umuchiana- } \\
\text { Ekwulobia }\end{array}$ & $\begin{array}{l}\text { N05.92925 } \\
0 \\
\text { E007.1257 } \\
2^{0}\end{array}$ & 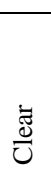 & 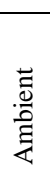 & $\begin{array}{l}\mathrm{Ni} \\
1\end{array}$ & $\begin{array}{l}\mathrm{Ni} \\
1\end{array}$ & $\begin{array}{l}\mathrm{Ni} \\
1\end{array}$ & $\begin{array}{l}5.8 \\
0\end{array}$ & 78.00 & $\begin{array}{l}39.0 \\
0\end{array}$ & Nil & Nil & $\begin{array}{l}\mathrm{Ni} \\
1\end{array}$ & 8.00 & $\begin{array}{l}13.0 \\
0\end{array}$ & 7.00 & 6.00 & - & $\begin{array}{l}8.0 \\
0\end{array}$ & $\begin{array}{l}0.0 \\
1\end{array}$ & 4.40 & $\begin{array}{l}0.0 \\
2\end{array}$ & $\begin{array}{l}0.0 \\
3\end{array}$ & $\begin{array}{l}\mathrm{Ni} \\
1\end{array}$ & - & 8.00 & 3.00 \\
\hline
\end{tabular}


Table 2: (Continued).

\begin{tabular}{|c|c|c|c|c|c|c|c|c|c|c|c|c|c|c|c|c|c|c|c|c|c|c|c|c|c|c|}
\hline & \multicolumn{6}{|c|}{ PHYSICAL PARAMETERS } & \multicolumn{18}{|c|}{ CHEMICAL PARAMETERS } & \multicolumn{2}{|c|}{$\begin{array}{l}\text { BACTERIOLOGIC } \\
\text { AL PARAMETERS }\end{array}$} \\
\hline & 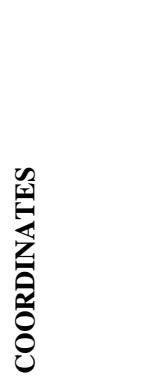 & 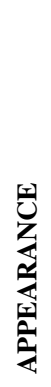 & 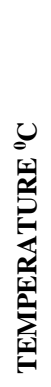 & 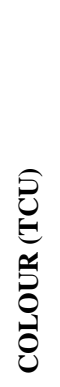 & 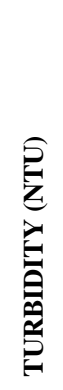 & 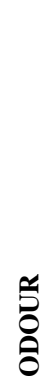 & : & 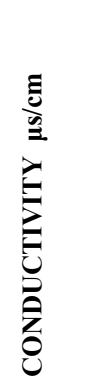 & 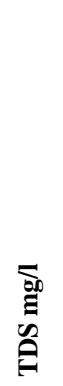 & 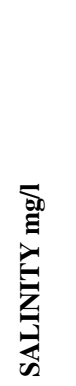 & 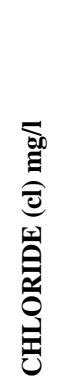 & 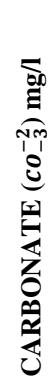 & 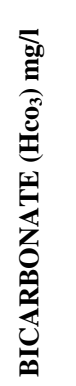 & 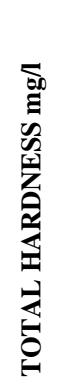 & 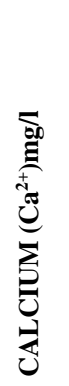 & 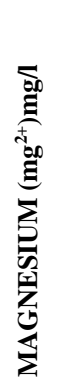 & 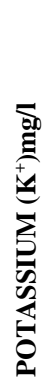 & 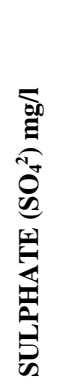 & 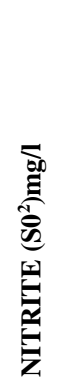 & 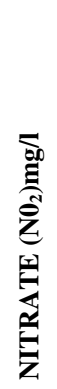 & 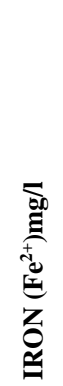 & 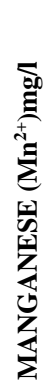 & 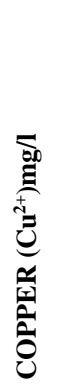 & 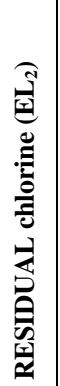 & & 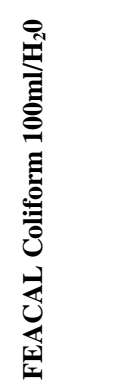 \\
\hline $\begin{array}{l}\text { 10. Ihokapala- } \\
\text { Ekwulobia }\end{array}$ & $\begin{array}{l}\text { N06.02993 }{ }^{0} \\
\text { E007.07469 } \\
0\end{array}$ & $\frac{\vec{\Xi}}{U}$ & 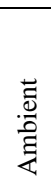 & $\begin{array}{l}\mathrm{Ni} \\
1\end{array}$ & $\begin{array}{l}\mathrm{Ni} \\
1\end{array}$ & $\begin{array}{l}\mathrm{Ni} \\
1\end{array}$ & 5.8 & 35.60 & $\begin{array}{l}17.8 \\
0\end{array}$ & Nil & Nil & $\begin{array}{l}\mathrm{Ni} \\
1\end{array}$ & 3.00 & 9.00 & 6.00 & 3.00 & - & $\begin{array}{l}4.0 \\
0\end{array}$ & Nil & $\begin{array}{l}7.0 \\
0\end{array}$ & 8.0 & $\begin{array}{l}0.1 \\
8\end{array}$ & $\begin{array}{l}0.0 \\
1\end{array}$ & - & 0.00 & 0.00 \\
\hline $\begin{array}{l}\text { 11. Ebele- } \\
\text { Achina }\end{array}$ & $\begin{array}{l}\text { N05 } 057^{1} 00.0 \\
1, \\
\text { E007 } 070.0^{1}\end{array}$ & 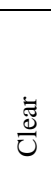 & $\frac{\overrightarrow{0}}{\stackrel{0}{0}}$ & $\begin{array}{l}\mathrm{Ni} \\
1\end{array}$ & $\begin{array}{l}\mathrm{Ni} \\
1\end{array}$ & $\begin{array}{l}\mathrm{Ni} \\
1\end{array}$ & $\begin{array}{l}6.4 \\
5\end{array}$ & $\begin{array}{l}142.5 \\
0\end{array}$ & $\begin{array}{l}71.4 \\
0\end{array}$ & $\begin{array}{l}16.5 \\
0\end{array}$ & $\begin{array}{l}10.0 \\
0\end{array}$ & $\begin{array}{l}\mathrm{Ni} \\
1\end{array}$ & $\begin{array}{l}79.0 \\
0\end{array}$ & $\begin{array}{l}86.0 \\
0\end{array}$ & $\begin{array}{l}52.0 \\
0\end{array}$ & $\begin{array}{l}34.0 \\
0\end{array}$ & - & $\begin{array}{l}2.0 \\
0\end{array}$ & $\begin{array}{l}0.0 \\
2\end{array}$ & $\begin{array}{l}4.4 \\
0\end{array}$ & 0.2 & $\begin{array}{l}0.0 \\
1\end{array}$ & Nil & - & 3.00 & 0.00 \\
\hline $\begin{array}{l}\text { 12. Umugama } \\
\text { Communit } \\
\text { y, Umuchu }\end{array}$ & $\begin{array}{l}\text { N05.92436 } \\
\text { E007.12789 }\end{array}$ & $\frac{\tilde{\Xi}}{U}$ & 苛 & $\begin{array}{l}\mathrm{Ni} \\
1\end{array}$ & $\begin{array}{l}\mathrm{Ni} \\
1\end{array}$ & $\begin{array}{l}\mathrm{Ni} \\
1\end{array}$ & $\begin{array}{l}6.1 \\
0\end{array}$ & 46.80 & $\begin{array}{l}23.4 \\
0\end{array}$ & Nil & Nil & $\begin{array}{l}\mathrm{Ni} \\
1\end{array}$ & $\begin{array}{l}13.0 \\
0\end{array}$ & $\begin{array}{l}34.0 \\
0\end{array}$ & $\begin{array}{l}20.0 \\
0\end{array}$ & $\begin{array}{l}14.0 \\
0\end{array}$ & - & $\begin{array}{l}1.0 \\
0\end{array}$ & Nil & $\begin{array}{l}1.0 \\
0\end{array}$ & 0.2 & Nil & Nil & - & 0.00 & 0.00 \\
\hline $\begin{array}{l}\text { 13. Agbaelu } \\
\text { Communit } \\
\text { y, Akpo }\end{array}$ & $\begin{array}{l}\text { N05.97018 } \\
\text { E007.10879 }\end{array}$ & $\stackrel{\vec{\varpi}}{U}$ & 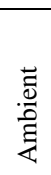 & $\begin{array}{l}\mathrm{Ni} \\
1\end{array}$ & $\begin{array}{l}\mathrm{Ni} \\
1\end{array}$ & $\begin{array}{l}\mathrm{Ni} \\
1\end{array}$ & $\begin{array}{l}5.2 \\
0\end{array}$ & 98.60 & $\begin{array}{l}49.1 \\
0\end{array}$ & 5.00 & 3.00 & $\begin{array}{l}\mathrm{Ni} \\
1\end{array}$ & $\begin{array}{l}12.0 \\
0\end{array}$ & $\begin{array}{l}62.0 \\
0\end{array}$ & $\begin{array}{l}37.0 \\
0\end{array}$ & $\begin{array}{l}25.0 \\
0\end{array}$ & - & Nil & $\begin{array}{l}0.0 \\
1\end{array}$ & $\begin{array}{l}8.0 \\
0\end{array}$ & Nil & Nil & Nil & - & 0.00 & 0.00 \\
\hline $\begin{array}{l}\text { 14. Umumilo } \\
\text { Communit } \\
\text { y-Umuchu }\end{array}$ & $\begin{array}{l}\text { N05.9434 } \\
\text { E00713057 }\end{array}$ & $\frac{\vec{\Xi}}{U}$ & 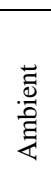 & $\begin{array}{l}\mathrm{Ni} \\
1\end{array}$ & $\begin{array}{l}\mathrm{Ni} \\
1\end{array}$ & $\begin{array}{l}\mathrm{Ni} \\
1\end{array}$ & $\begin{array}{l}6.1 \\
0\end{array}$ & 72.50 & $\begin{array}{l}36.0 \\
0\end{array}$ & Nil & Nil & $\begin{array}{l}\mathrm{Ni} \\
1\end{array}$ & $\begin{array}{l}92.0 \\
0\end{array}$ & $\begin{array}{l}58.0 \\
0\end{array}$ & $\begin{array}{l}34.0 \\
0\end{array}$ & $\begin{array}{l}24.0 \\
0\end{array}$ & - & $\begin{array}{l}2.0 \\
0\end{array}$ & Nil & $\begin{array}{l}2.6 \\
0\end{array}$ & $\begin{array}{l}0.2 \\
0\end{array}$ & $\begin{array}{l}0.0 \\
1\end{array}$ & $\begin{array}{l}0.0 \\
1\end{array}$ & - & 0.00 & 0.00 \\
\hline
\end{tabular}


Table 2: (Continued)

\begin{tabular}{|c|c|c|c|c|c|c|c|c|c|c|c|c|c|c|c|c|c|c|c|c|c|c|c|c|c|c|}
\hline & \multicolumn{6}{|c|}{$\begin{array}{l}\text { PHYSICAL PARAMETERS } \\
\end{array}$} & \multicolumn{18}{|c|}{ CHEMICAL PARAMETERS } & \multicolumn{2}{|c|}{$\begin{array}{c}\text { BACTERIOLOGICA } \\
\text { L PARAMETERS }\end{array}$} \\
\hline & 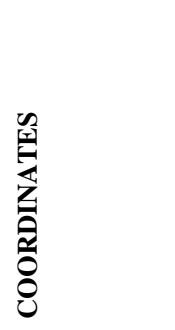 & 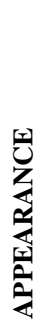 & 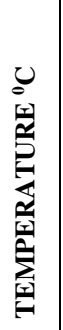 & 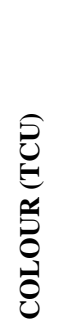 & 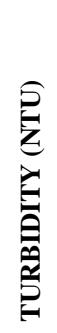 & $\begin{array}{l}\text { 응 } \\
\text { 。ิ }\end{array}$ & 到 & 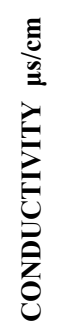 & 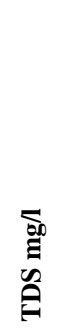 & 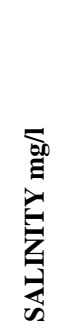 & 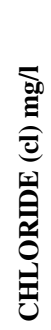 & 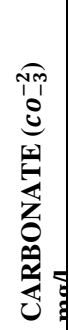 & 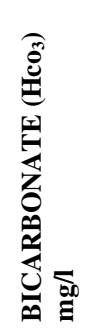 & 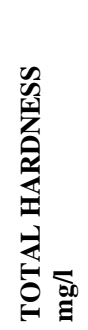 & 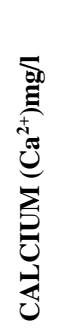 & 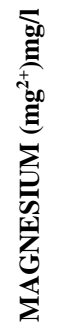 & 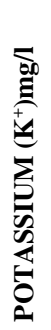 & 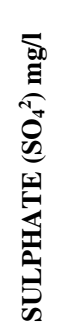 & 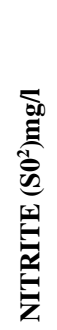 & 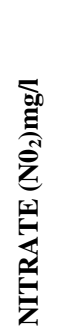 & 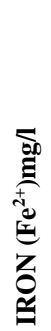 & 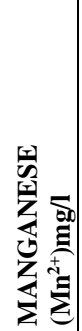 & 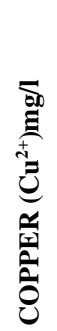 & 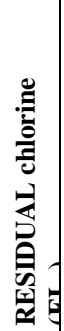 & 递 & 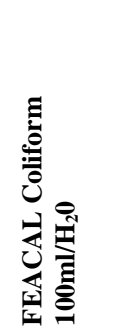 \\
\hline $\begin{array}{l}\text { 15. Special } \\
\text { Education } \\
\text { Centre } \\
\text { Umuchu }\end{array}$ & $\begin{array}{l}\text { N05.9295 } \\
\text { E007.12572 }\end{array}$ & 离 & 善 & $\begin{array}{l}\mathrm{Ni} \\
1\end{array}$ & $\begin{array}{l}\mathrm{Ni} \\
1\end{array}$ & $\begin{array}{l}\mathrm{Ni} \\
1\end{array}$ & $\begin{array}{l}6.1 \\
0\end{array}$ & $\begin{array}{l}72.5 \\
0\end{array}$ & $\begin{array}{l}36,0 \\
0\end{array}$ & $\begin{array}{l}\mathrm{Ni} \\
1\end{array}$ & $\begin{array}{l}\mathrm{Ni} \\
1\end{array}$ & $\begin{array}{l}\mathrm{Ni} \\
1\end{array}$ & $\begin{array}{l}92.0 \\
0\end{array}$ & $\begin{array}{l}58.0 \\
0\end{array}$ & $\begin{array}{l}34.0 \\
0\end{array}$ & $\begin{array}{l}24.0 \\
0\end{array}$ & - & $\begin{array}{l}2.0 \\
0\end{array}$ & Nil & $\begin{array}{l}2.6 \\
0\end{array}$ & $\begin{array}{l}0.2 \\
0\end{array}$ & $\begin{array}{l}0.0 \\
1\end{array}$ & $\begin{array}{l}0.0 \\
1\end{array}$ & - & 0.00 & 0.00 \\
\hline $\begin{array}{l}\text { 16. Obeagu- } \\
\text { Ezinifite }\end{array}$ & $\begin{array}{l}\text { N05.98227, } \\
\text { E007.06930 }\end{array}$ & $\frac{\vec{\Xi}}{U}$ & $\begin{array}{l}\overrightarrow{\overline{ \pm}} \\
\text { है } \\
\text { हो }\end{array}$ & $\begin{array}{l}\mathrm{Ni} \\
1\end{array}$ & $\begin{array}{l}\mathrm{Ni} \\
1\end{array}$ & $\begin{array}{l}\mathrm{Ni} \\
1\end{array}$ & $\begin{array}{l}5.8 \\
0\end{array}$ & $\begin{array}{l}22.3 \\
0\end{array}$ & $\begin{array}{l}11.3 \\
0\end{array}$ & $\begin{array}{l}\mathrm{Ni} \\
1\end{array}$ & $\begin{array}{l}\mathrm{Ni} \\
1\end{array}$ & $\begin{array}{l}\mathrm{Ni} \\
1\end{array}$ & 7.00 & $\begin{array}{l}15.0 \\
0\end{array}$ & 5.00 & $\begin{array}{l}10.0 \\
0\end{array}$ & - & $\begin{array}{l}7.0 \\
0\end{array}$ & $\begin{array}{l}0.0 \\
1\end{array}$ & $\begin{array}{l}1.7 \\
6\end{array}$ & $\begin{array}{l}0.0 \\
3\end{array}$ & Nil & Nil & - & 0.00 & 0.00 \\
\hline 17. Ndiowu & $\begin{array}{l}\text { N06.32.112 }{ }^{1}, \\
\text { E00780.002 }\end{array}$ & 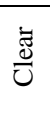 & 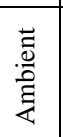 & $\begin{array}{l}\mathrm{Ni} \\
1\end{array}$ & $\begin{array}{l}\mathrm{Ni} \\
1\end{array}$ & $\begin{array}{l}\mathrm{Ni} \\
1\end{array}$ & $\begin{array}{l}5.7 \\
0\end{array}$ & $\begin{array}{l}66.0 \\
0\end{array}$ & $\begin{array}{l}35.0 \\
0\end{array}$ & $\begin{array}{l}\mathrm{Ni} \\
1\end{array}$ & $\begin{array}{l}\mathrm{Ni} \\
1\end{array}$ & $\begin{array}{l}\mathrm{Ni} \\
1\end{array}$ & $\begin{array}{l}40.0 \\
0\end{array}$ & $\begin{array}{l}65.0 \\
0\end{array}$ & $\begin{array}{l}41.0 \\
0\end{array}$ & $\begin{array}{l}22.0 \\
0\end{array}$ & - & $\begin{array}{l}1.0 \\
0\end{array}$ & Nil & $\begin{array}{l}1.0 \\
0\end{array}$ & Nil & Nil & Nil & - & 1.00 & 0.00 \\
\hline 18. Ekwulumili & $\begin{array}{l}\mathrm{N}^{0} 5^{0} 58401^{1}, \\
\text { E007 } \\
1\end{array}$ & $\frac{\vec{\Xi}}{U}$ & 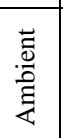 & $\begin{array}{l}\mathrm{Ni} \\
1\end{array}$ & $\begin{array}{l}\mathrm{Ni} \\
1\end{array}$ & $\begin{array}{l}\mathrm{Ni} \\
1\end{array}$ & $\begin{array}{l}5.5 \\
0\end{array}$ & $\begin{array}{l}43.0 \\
0\end{array}$ & $\begin{array}{l}21.0 \\
0\end{array}$ & $\begin{array}{l}\mathrm{Ni} \\
1\end{array}$ & $\begin{array}{l}\mathrm{Ni} \\
1\end{array}$ & $\begin{array}{l}\mathrm{Ni} \\
1\end{array}$ & $\begin{array}{l}20.0 \\
0\end{array}$ & $\begin{array}{l}40.0 \\
0\end{array}$ & $\begin{array}{l}25.0 \\
0\end{array}$ & $\begin{array}{l}15.0 \\
0\end{array}$ & - & Nil & $\begin{array}{l}0.0 \\
2\end{array}$ & $\begin{array}{l}5.0 \\
0\end{array}$ & $\begin{array}{l}0.0 \\
1\end{array}$ & Nil & Nil & - & 1.00 & 0.00 \\
\hline 19. Ndiopaeze & $\begin{array}{l}\text { N06 } 00.238^{1} \text {. } \\
\text { E007 } \\
1\end{array}$ & $\frac{\vec{\Xi}}{U}$ & 离 & $\begin{array}{l}\mathrm{Ni} \\
1\end{array}$ & $\begin{array}{l}\mathrm{Ni} \\
1\end{array}$ & $\begin{array}{l}\mathrm{Ni} \\
1\end{array}$ & $\begin{array}{l}5.7 \\
0\end{array}$ & $\begin{array}{l}50.0 \\
0\end{array}$ & $\begin{array}{l}24.0 \\
0\end{array}$ & $\begin{array}{l}\mathrm{Ni} \\
1\end{array}$ & $\begin{array}{l}\mathrm{Ni} \\
1\end{array}$ & $\begin{array}{l}\mathrm{Ni} \\
1\end{array}$ & $\begin{array}{l}17.0 \\
0\end{array}$ & $\begin{array}{l}31.0 \\
0\end{array}$ & $\begin{array}{l}25.0 \\
0\end{array}$ & $\begin{array}{l}20.0 \\
0\end{array}$ & - & Nil & $\begin{array}{l}0.0 \\
1\end{array}$ & $\begin{array}{l}1.0 \\
0\end{array}$ & Nil & Nil & Nil & - & 0.00 & 0.00 \\
\hline 20. Oko & $\begin{array}{l}\text { N06 } 25.3201 \\
\text { E007041.321 } \\
1\end{array}$ & $\frac{\vec{d}}{U}$ & 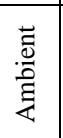 & $\begin{array}{l}\mathrm{Ni} \\
1\end{array}$ & $\begin{array}{l}\mathrm{Ni} \\
1\end{array}$ & $\begin{array}{l}\mathrm{Ni} \\
1\end{array}$ & $\begin{array}{l}6.0 \\
0\end{array}$ & $\begin{array}{l}70.0 \\
0\end{array}$ & $\begin{array}{l}33.0 \\
0\end{array}$ & $\begin{array}{l}\mathrm{Ni} \\
1\end{array}$ & $\begin{array}{l}\mathrm{Ni} \\
1\end{array}$ & $\begin{array}{l}\mathrm{Ni} \\
1\end{array}$ & $\begin{array}{l}30.0 \\
0\end{array}$ & $\begin{array}{l}26.0 \\
0\end{array}$ & $\begin{array}{l}30.0 \\
0\end{array}$ & $\begin{array}{l}17.0 \\
0\end{array}$ & - & Nil & $\begin{array}{l}0.0 \\
1\end{array}$ & $\begin{array}{l}1.0 \\
0\end{array}$ & Nil & $\begin{array}{l}0.0 \\
2\end{array}$ & Nil & - & 0.00 & 0.00 \\
\hline
\end{tabular}


Statistical methods were used to obtain the mean, range, variance and standard deviation for the $\mathrm{pH}$ and major anions and cations found in water samples of the study area. The mean $\mathrm{pH}$ is 5.8 with range of 0.8 , variance of 0.07 and standard deviation of 0.26 . The mean value for conductivity is $67.13 \mathrm{uS} / \mathrm{cm}$, range is $104.9 \mathrm{uS} / \mathrm{cm}$, variance is $844 \mathrm{uS} / \mathrm{cm}$ and standard deviation is $29.1 \mathrm{uS} / \mathrm{cm}$. Calcium and Magnesium ions gave mean values of $2.4 \mathrm{mg} / 1$ and $15.8 \mathrm{mg} / 1$, range of $33 \mathrm{mg} / \mathrm{l}$ and $21 \mathrm{mg} / \mathrm{l}$, variance of $87.3 \mathrm{mg} / \mathrm{l}$ and $39.3 \mathrm{mg} / \mathrm{l}$ and standard deviation of $9.3 \mathrm{mg} / \mathrm{l}$ and $6.3 \mathrm{mg} / \mathrm{l}$ respectively. The mean value for sodium is $22.0262 \mathrm{mg} / \mathrm{l}$, range is $28.842 \mathrm{mg} / \mathrm{l}$, variance is $70.8215 \mathrm{mg} / \mathrm{l}$ and standard deviation of $8.4 \mathrm{mg} / \mathrm{l}$. Potassium value is $8.83 \mathrm{mg} / \mathrm{l}$ for mean, $8.29 \mathrm{mg} / \mathrm{l}$ for range, $5.5099 \mathrm{mg} / \mathrm{l}$ for variance and $2.3 \mathrm{mg} / \mathrm{l}$ for standard deviation. The $\mathrm{Ca}^{++} \mathrm{Mg}++, \mathrm{Na}+$ and $\mathrm{K}+$ cations analysed fall within the recommended standard for potable water World Health Organisation (WHO), 2006. The mean sulfate concentration is $1.75 \mathrm{mg} / \mathrm{l}$ range is $6 \mathrm{mg} / \mathrm{l}$, variance is $2.7 \mathrm{mg} / \mathrm{l}$ and standard deviation is $1.6 \mathrm{mg} / \mathrm{l}$. Chloride value for mean is $4.99 \mathrm{mg} / \mathrm{l}$ range is $5.82 \mathrm{mg} / \mathrm{l}$, variance is $2.72 \mathrm{mg} / \mathrm{l}$ and standard deviation is $1.65 \mathrm{mg} / \mathrm{l}$. The bicarbonate ion has a mean value of $35.65 \mathrm{mg} / 1$ range is $72 \mathrm{mg} / \mathrm{l}$, variance is $392 \mathrm{mg} / \mathrm{l}$ and standard deviation is $20 \mathrm{mg} / \mathrm{l}$. A mean value of $36.7 \mathrm{mg} / \mathrm{l}$ for total hardness was obtained, range is $54 \mathrm{mg} / \mathrm{l}$, variance is $238 \mathrm{mg} / \mathrm{l}$ and standard deviation is $15.4 \mathrm{mg} / \mathrm{l}$. Mean of $35.06 \mathrm{mg} / \mathrm{l}$ was obtained for Total dissolve solids. (TDS), range of $52.4 \mathrm{mg} / \mathrm{l}$, variance of $205 \mathrm{mg} / \mathrm{l}$ and standard deviation of $14.3 \mathrm{mg} / \mathrm{l}$. Which all fall within the recommended standard for potable water World Health Organization (WHO), 2006 Guidelines.
Chemical Analysis: In order to have a more defined and clearer picture of the chemistry of the water under study, the data acquired were further processed using the piper, Durov, Piper and Schoeller semi log diagrams

\section{Piper diagram}

The major ionic species in most natural waters are $\mathrm{Na}, \mathrm{K}, \mathrm{Ca}$, $\mathrm{Mg} \mathrm{Cl}, \mathrm{CO} 3, \mathrm{HCO} 3$, and SO4. This trilinear diagram showed the percentage composition of three ions. $\mathrm{Na}$ and $\mathrm{K}$, the major cations were grouped; there are also three groups of the major anions (Fig. 5). Since all three constituent groups are present, the analyses fell in the interior of the field. The diamondshaped field between the two triangles was then used to represent the composition of water with respect to both cations and anions. The cation point was projected onto the diamond-shaped field parallel to the side of the triangle labeled magnesium and the anion point was similarly projected parallel to the side of the triangle labeled sulfate. The intersection of the two lines was plotted as a point on the diamond-shaped field. This exercise revealed that the type of water present is calcium, magnesium and bicarbonate types.

\section{Schoeller semilogarithmic Diagram}

Schoeller Diagram (Freeze and Cherry, 1979), which was used to verify the results obtained from the Piper diagram, allowed us to make a visual comparison of the compositions of different waters, (Fig. 6). Sodium and Potassium were grouped and the result showed that magnesium is the dominant type followed by calcium and then, bicarbonate.
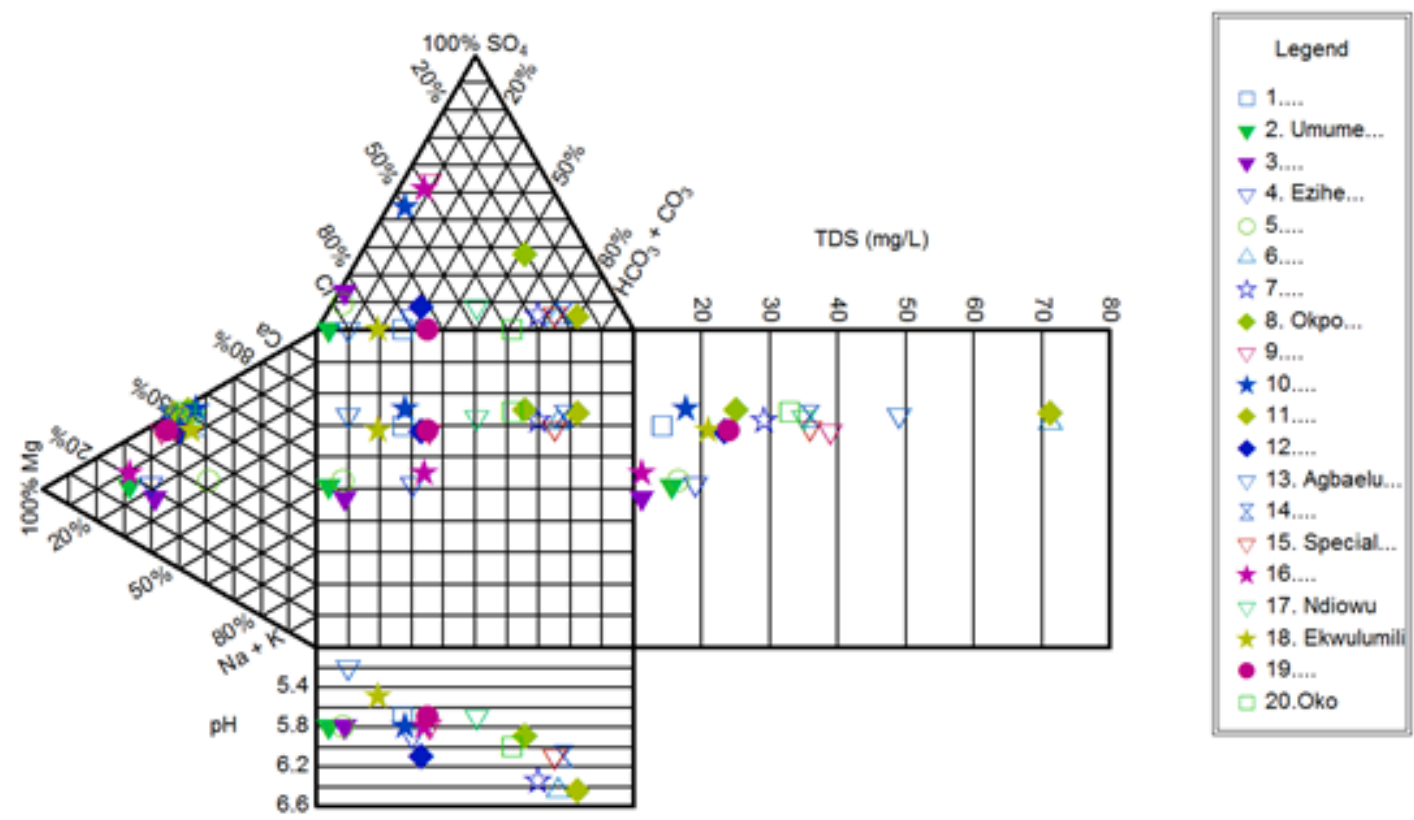

Figure 5 Graphical Illustration of Hydrogeochemical Data on the Piper Diagram. 

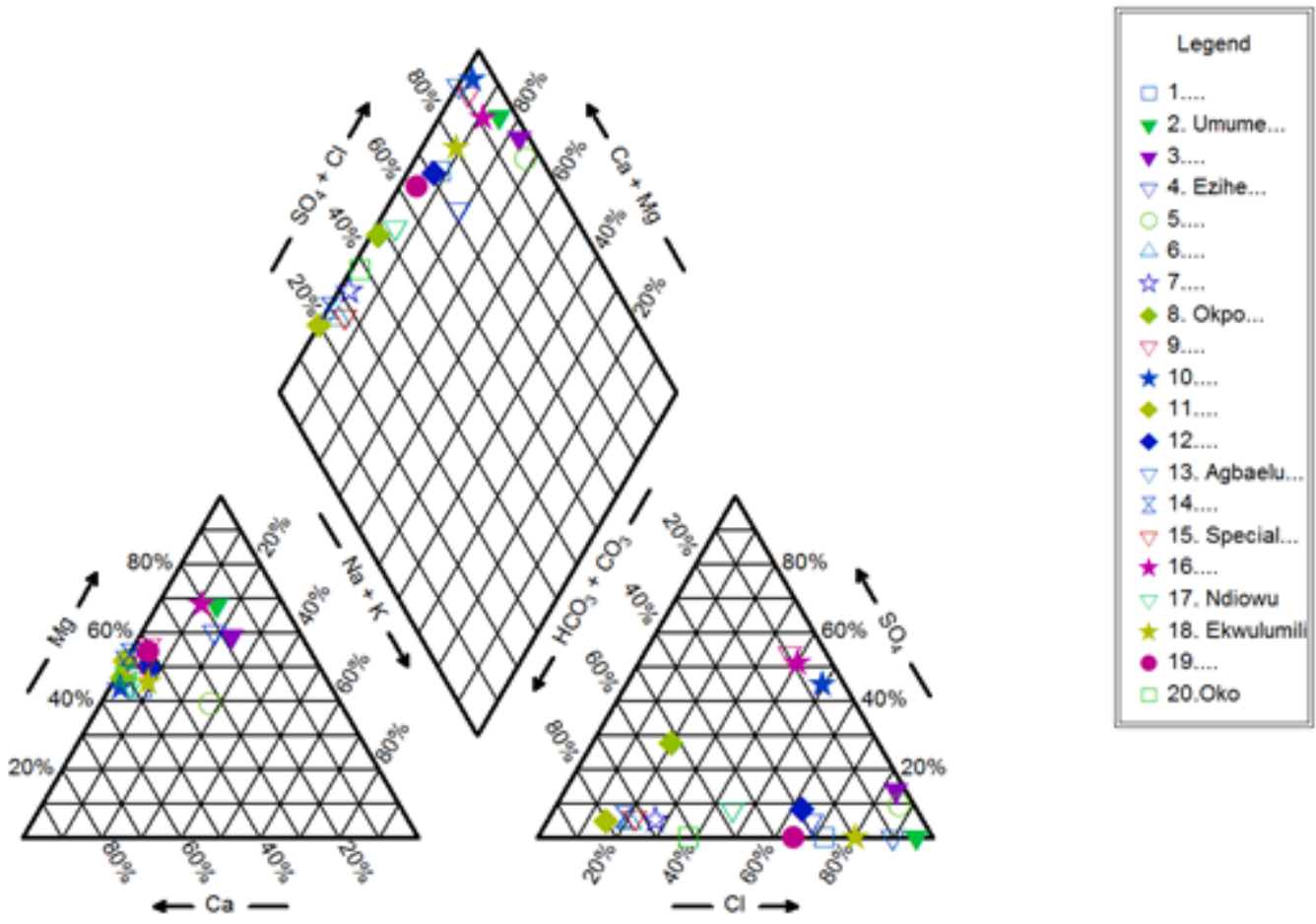

Figure 5: (Continued).

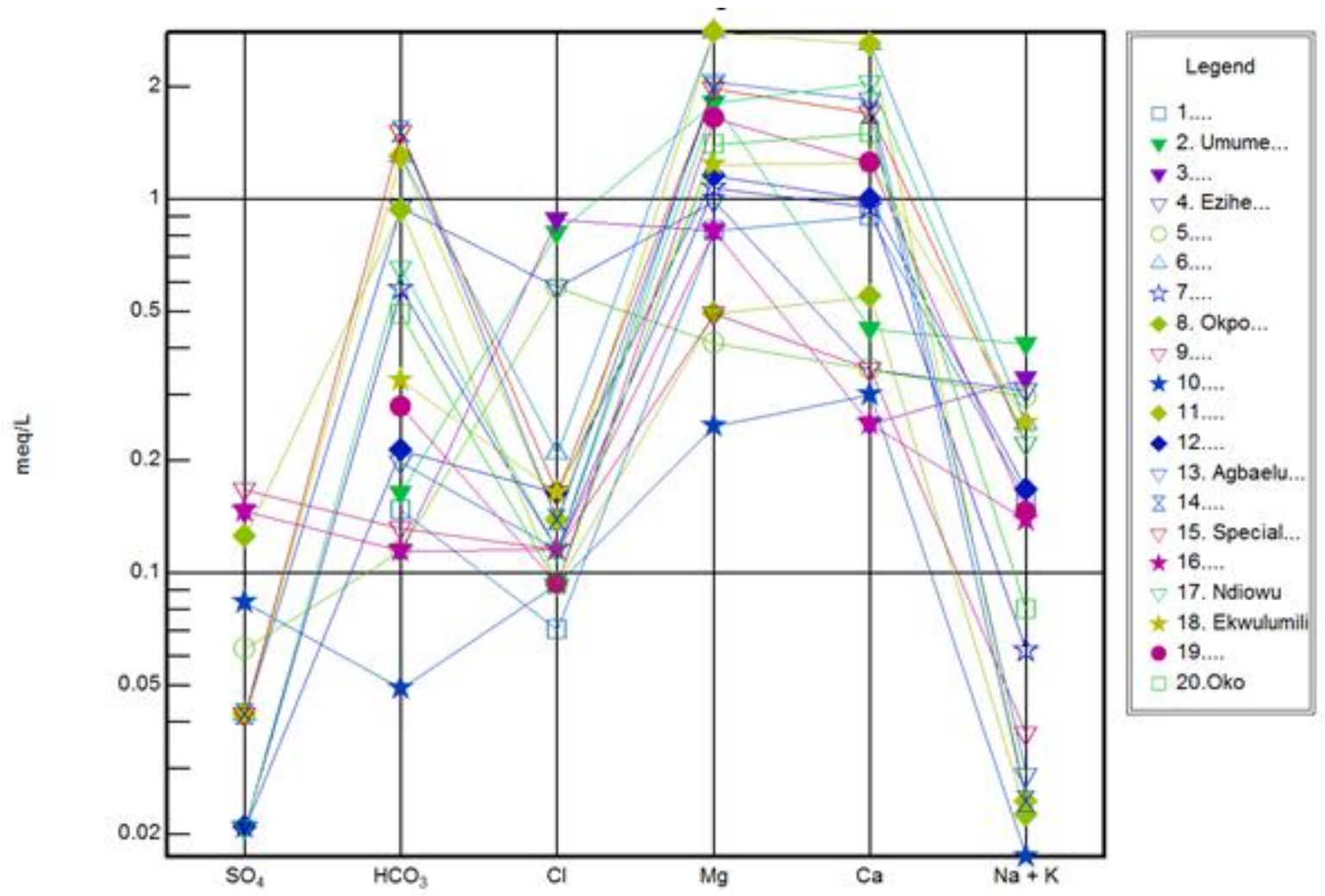

Figure 6: Schoeller Diagram. 
Table 3: Nigeria Standard for Drinking Water quality (adopted from Standards Organization of Nigeria, 2007).

\begin{tabular}{|c|c|c|c|c|}
\hline & PARAMETER & UNIT & $\begin{array}{l}\text { MAX. } \\
\text { PERMISSIBLE }\end{array}$ & HEALTH IMPACT \\
\hline \multirow{7}{*}{$\begin{array}{l}\text { PHYSICAL } \\
\text { PARAMETER }\end{array}$} & Colour & TCU & 15 & None \\
\hline & Odour & - & Unobjectional & None \\
\hline & Taste & - & Unobjectional & None \\
\hline & Temperature & ${ }^{0} \mathrm{C}$ & Ambient & None \\
\hline & Turbidity & NTU & 5 & None \\
\hline & Conductivity & Us/cm & 1000 & None \\
\hline & Copper $\left(\mathrm{Cu}^{2+}\right)$ & $\mathrm{Mg} / \mathrm{L}$ & 1.00 & Gastrointestinal disorder \\
\hline \multirow{11}{*}{$\begin{array}{l}\text { CHEMICAL } \\
\text { PARAMETERS }\end{array}$} & Chloride (CL-) & $\mathrm{Mg} / \mathrm{L}$ & 250 & None \\
\hline & Hardness & $\mathrm{Mg} / \mathrm{L}$ & 150 & None \\
\hline & Iron $\mathrm{fe}^{+2}$ & $\mathrm{Mg} / \mathrm{L}$ & 0.30 & None \\
\hline & Magnesium & $\mathrm{Mg} / \mathrm{L}$ & 0.20 & Consumer acceptability \\
\hline & Manganese & $\mathrm{Mg} / \mathrm{l}$ & 0.20 & Neurological disorder \\
\hline & Nitrate $\left(\mathrm{NO}_{3}\right)$ & $\mathrm{Mg} / \mathrm{L}$ & 50.00 & $\begin{array}{l}\text { Cyanosi and Asphyxia(blue baby } \\
\text { syndrome)in infants under } 3 \text { months }\end{array}$ \\
\hline & Nitrite $\left(\mathrm{NO}_{2}\right)$ & $\mathrm{Mg} / \mathrm{L}$ & 0.20 & $\begin{array}{l}\text { Cyanosi and Asphyxia(blue baby } \\
\text { syndrome)in infants under } 3 \text { months }\end{array}$ \\
\hline & $\mathrm{pH}$ & - & $6.5-8.5$ & None \\
\hline & Sodium $(\mathrm{Na})$ & $\mathrm{Mg} / \mathrm{L}$ & 200.00 & None \\
\hline & Sulphate $\left(\mathrm{SO}_{4}\right)$ & $\mathrm{Mg} / \mathrm{L}$ & 100.00 & None \\
\hline & TDS & $\mathrm{Mg} / \mathrm{L}$ & 500.00 & None \\
\hline \multirow[t]{2}{*}{ MICROBIOLOGICAL } & Total Coliform & $\mathrm{Cfu} / \mathrm{mlo}$ & 10.00 & Indication of faecal contamination \\
\hline & Faecal coliform & $\mathrm{Cfu} / 100 \mathrm{ml}$ & 0.00 & $\begin{array}{l}\text { Indication of recent faecal } \\
\text { contamination }\end{array}$ \\
\hline
\end{tabular}

\section{DISCUSSION}

Water quality analyses were carried out on some water samples collected from the study areas. In scope of this study can be divided into three domains. Microbial contamination refers to the presence of disease causing (or pathogene) microbes. Which are generally introduced to water sources by contact with faecal material. Chemical contamination refers to metals, organic compounds and other chemicals that present potential health risk. Physical contamination refers to condition relating to water physical condition, for example colour, odour, temperature and turbidity (cloudiness resulting from the presence of small particles like pieces of soil).

Water Quality analyses for physical parameter showed that the temperature was ambient, the water were colourless, odourless, and tasteless which was within the World Health Organization (WHO, 2006) permissible limit. The major chemical species in most natural waters are Sodium, potassium, magnesium, calcium, chloride, carbonates, bicarbonates and sulphates. For chemical parameters the $\mathrm{pH}$ ranged from 5.20 at Ihuowelle Igboukwu to 6.80, at Ebele Achina(highest), total dissolved solids (TDS) from $11.20 \mathrm{mg} / 1$ at Ezinifite to $71.40 \mathrm{mg} / \mathrm{l}$ at Achina ,turbidity nil, major cations and anions, calcium $\left(\mathrm{Ca}^{2+}\right)$ ranged from $5.00 \mathrm{mg} / \mathrm{l}$ at Okwuchiezeada Ezinifite to $42.00 \mathrm{mg} / \mathrm{l}$ at Ndiowu,magnesium $\left(\mathrm{Mg}^{2+}\right)$ ranged from $0.01 \mathrm{mg} / \mathrm{l}$ at Uga to $0.18 \mathrm{mg} / 1$ in Ekwulobia,sulphate was not detected, iron ranged from $0.02 \mathrm{mg} / 1$ to $0.35 \mathrm{mg} / \mathrm{l}$ in Igboukwu, Umueze Uga $(0.35 \mathrm{mg} / \mathrm{l})$, manganese, copper and residual chlorine were not detected whileBicarbonate $\left(\mathrm{HCO}_{3}\right)$ ranged from $7.00 \mathrm{mg} / 1$ at Ezinifite to $58.00 \mathrm{mg} / \mathrm{l}$ at $\mathrm{Uga}$ and Sulphate $\left(\mathrm{SO}_{4}{ }^{2}\right)$ ranged from $0.00 \mathrm{mg} / \mathrm{l}$ at Igboukwu to $7.00 \mathrm{mg} / \mathrm{l}$ at Ekwulobia .
Bacteriological result showed that total coliform and feacel coliform is zero. The water quality parameters tested fall within the World Health Organization (WHO, 2006) guidelines, desirable, permissible, acceptable limits and Nigeria Standard for Drinking Water Quality (NSDWQ, 2011) apart from the $\mathrm{pH}$ values which fall below the World Health Organization standard in some areas, though $\mathrm{pH}$ do not pose any health risk in water. The water quality of the groundwater resources in the study area is, therefore described as good. According to the Standard Organization of Nigeria, all water sources intended for human consumption shall comply with Nigeria Standard for Drinking Water Quality (NSDWQ, 2011) as shown in Table 3.

The graphical illustration of the hydrogeochemical analysis carried out using the Piper diagram, Stiff diagram, Shoeller semi logarithm diagram and Durov diagram all showed that "Calcium and Bicarbonate" were the main type of water in the study area and "Magnesium and Bicarbonate" were the additional type water.

\section{REFERENCES}

Ajayi, (1987). Politics of rural development in Nigeria, pointing out that the effects of industrializing rural areas of the country is a minus on the health of the community. Vol 4 pp 69.

Cherry, J. A. (1999). Contamination incidents occurring from industrial waste, landfill, leachates, petroleum products, organic wastes as well as chlorides radioactive wastes pp. 42-60.

De Rooy, C., Kamfort, M., and Sambile, O. (1986). An Empirical Hydrogeological Model for

Dunham et al.,Andreasenet al., Salt (1992). Sulphur, an Important Constituent of Coals, major source of pollution from coal-fired plants. Vol. 8 pp 59-62.

Edington, G.M. and Mcheen, C.M.U. (1965) A Cancer rate survey in Ibadan, Western Nigeria. Bir. J. Cancer, Vol. 19 pp 471-481. 
Egboka and Ezeonu (1990).Nitrate and Nitrite pollution and contamination in parts of southeastern Nigeria.Afric.Earth sci. J. 3 pp 432.

Egboka and Onyebueke, (1990).Environmental hazards occurring from the discharge of industrial wastes, acid and iron-rich leachates from coal mines into the hydrogeological environment. Pp. 80-85.

Egboka, B.C.E and Okpoko, I..E, (1989). Lead poisoning and guinea worm infestation in lead processing area of Abakaliki Nigeria pp. 67, 70

Egboka, B. C. E. \&Nwankwo, G. I. (1985). The hydrogeological and geotechnical parameters as agents of gully type of erosion in the rainforest belt of Nigeria. Afric. Earth Sci. J. 3, pp 417-425.

Egboka, B. C. E. (1993). The Raging War.God's Time Printing \& Publishing, Awka. P223

Egboka, B. C. E., Nfor, B. N and Banlanjo, E.W. (2006).Water budget analysis of Agulu Lake in Anambra State, Nigeria.J.App. Sci. Environ. Mgt. 10 (3), pp 27-30

Egboka, B. C. E., Nwankor, G. I. and Orajaka, T. P. (1990).Investigations of Paleo and Neotectonics in Gully Erosion-prone Areas of Southeastern Nigeria.Natural Hazards. Vol. 3, pp. 219-231.

Ezeigbo, (1991).Efficiency of water resources management in Nigeria pp. 6-10.

Ezeigbo, H. I. (1987). Quality of water resources in Anambra State, Nigeria.Journal of Mining and Geology. No. 23, Vol. 1 and 2, pp. 97-103.

Ezeigbo, H. I., Ozoko, D. C. (1989). An Evaluation of the Water Resources of Nsukka and environs, Anambra State, Nigeria.Water Resources Journal of Nigeria Association of Hydrogeologists. Vol. No. 2. pp. 20-25.

Ezenwa, S. O. (1996). The Relationship between Total Dissolved Solids (TDS) and Total Dissolved Iron (TDI) in aquifers of Southern Anambra Basin.Water Resources Journal of Nigeria Association of Hydrogeologists. Vol. 7 No. 1.Pp 31-42.

Fader and Eiraimah (1989).The injection of environmental impact assessment (EIA) into the environmental laws.

Falconer, J. D. (1911).The Geology and Geography of Northern Nigeria.Macmillan London, 295, 1911. Universal Journal of Geoscience 2(3): 93-102.

Fetter, C.W. Jnr. (2001). Applied Hydrogeology, $4^{\text {th }}$ Edition

Freeze, A.R., and Cherry, J.A.,1979, Groundwater (1st ed.), Upper Saddle River, N.J.,

Pearson Education, Inc., p. 247-253.

Groundwater Prospecting.Nature and Resources, Vol. XXX 1. No.

Heath, R. C. (1983). Basic Groundwater Hydrolog, U. S Geological Survey water supply paper 2220

Johnson, (1982).Survey of manufacturing areas in Nigeria cities.

Lindorff, (1979). Groundwater pollution incidents in parts of USA, pp. 32-36

Lorna Fewtrell and Jamie Bartram. (2001). W.H.O.Water quality: Guidelines, standards and health

Assessment of risk and risk management for water-related infectious disease. IWA Publishing. ISBN: 924154533X.

Meckelvey, (2005). Effects of Industrialization in parts of Japan U.S. Geological Survey Water Supply paper pp. 106.

Narasimha Prasad, N. B., Shivraj, P.V. \&Jegatheesan, M.S., (2007).Evaluation of the groundwater development prospects in Kadalundi river basin. J. Geolo. Soc. India, 69, pp 1103-11 10

Nfor, B. N. (2003). Sedimentary Facies and the Diagnostic Characteristics for the Campanian-Eocene Anambra Basin; Unpublished Ph.D thesis submitted to the Department of Geological Sciences, NnamdiAzikiwe University, Awka. 236P.
Nfor, B. N., Olabaniyi, S. B, and Ogala, J. E. (2007).Extent and Distribution of Groundwater Resources in parts of Anambra State, Southeastern Nigeria. Journal of Appl. Sci. Environ. Manage. Vol. 11 (2), 215-221

Nwajide, C. S. (1979). A Lithostratigraphic Analysis of Nanka Sands, Southern Nigeria. Journal of Mineral Geology in Nigeria PP. 103-108

Nwajide, S. C. \&Hoque, M. (1979). Gully processes in south-eastern Nigeria. The Nig. Field J. 44(2), pp 64-74.

Oformah, J. C., Ezeigbo, H. I. (1997). Hydrogeological Evaluation of the Anambra River Basin, Southeastern Nigeria.Water Resources Journal of Nigeria Association of Hydrogeologists. Vol. S.Nos. land 2. pp 18-26

Ogbukagu, I. M. (1976).Soil erosion in the northern part of Awka-Orlu uplands, Nig. J. Min. and Geol. 13, pp 6-19.

Okoro, E. I., Egboka, B. C. E., Onwuemesi, A. G. (2010). Evaluation of the aquifer characteristic of Nanka Sands using hydrogeological method in combination with Vertical Electrical Sounding (VES).J. Appl. Sci. Environ. Mgt. Vol. 14 ( 2) p p 5 - 9

Okpoko and Egboka (1986).Detailed hydrological and environmental Studies of Awka and Njikoka Local Government Areas.

Onwuemesi, (1990).Hydrogeological and geotechnical investigations of the Ajali Sandstone in Nsukka and environs with reference to groundwater resources and gully erosion problems.Pp 60-66.

Onwuemesi, A. G. \&Egboka, B. C. E. (2006).2-D Polynomial curve fitting techniques on watertable and hydraulic gradient estimations in pats of Anambra Basin, Southeastern Nigeria.Natural and Applied Sci. Journal 7 (1\&2), pp6-13.

Onwuemesi, A. G., Olaniyan, I. O. (1996). Hydrogeological Investigation of parts of Anambra State, Nigeria.Water Resources Journal of Nigeria Association of Hydrogeologists. Vol. 8. Nos. land 2. pp 56-61.

Rainwater, F. H. \& Thatcher, L. L. (1960).Methods for Collection and Analysis of Water Samples, U.S. Geological Survey Water-Supply Paper.Pp 1454, 301.

Reyment, R. A. (1965). Aspects of the Geology of Nigeria.University of Ibadan Press. 133P.

Sack, (1973).Pollutants from industrial emission poses a serious environmental hazard to human life. $6^{\text {th }}$ ed, U.S Geological Survey professional paper. Pp. 330-f, 68.

Sacks, L. A., Swancar, A. \& Lee, T. M. (1998).Estimating GroundWaterExchange with Lakes Using Water-Budget and Chemical Mass-Balance Approaches for Ten Lakes in Ridge Areas of Polk and Highlands Counties, Florida, U.S. Geological Survey Water Resources investigations Report 98-4133, Tallahassee, FL.

Standard Organization of Nigeria. (2007). Nigerian Standard for Drinking Water Quality. NIS 554: 2007. PP. 30

Tattam and Bain, (1944).The stratigraphy of Eastern Nigeria, biostratigraphy of Eastern Nigeria using ammonites, pelecypods and for aminitera as index fossils.

Ume and Uchegbu, Colleman, (1977). The bench mark data base collected in trava process of implementing the EIA, determining the accuracy prediction and efficiency of mitigation measures Vol 6 pp 106.

White, D. E., Hem, J.D. and Warring, G.S.., (1963).Data of Geochemistry-Chemical Composition of Subsurface Waters, 6th ed., U.S. Geological Survey Professional Paper.Pp 440-F, 67.

\section{$\underline{\text { How to cite this article }}$}

Ajanobi, C.C., Nfor, B.N., Okpoko, E.I. and Anene, C.Z. (2018). Water Quality Evaluation of Aquifers around Igboukwu and Environs, Southeastern Nigeria. Tropical Journal of Applied Natural Sciences, 2(2): 85-96. Doi: https://doi.org/10.25240/TJANS.2018.2.2.12. 